\title{
COMMISSION 16: PHYSICAL STUDY OF PLANETS AND BATELLITES (ETUDE PHYSIQUE DES PLANETES ET SATELLITES)
}

\author{
PRESIDENT: André Brahic \\ VICE-PRESIDENTS: David Morrisson, Michael Marov
}

\author{
SECRETARY: David Cruikshank
}

ORGANIZING COMMITTEE: Jean Loup Bertaux, Joseph Burns, Chen Dahoan, Merton Davies, Catherine De Bergh, Thérèse Encrenaz, Daniel Gautier, Harold Masursky, Dennis Matson, V.I. Moroz, Tobias Owen, vladislav shevshenko, Bradford smith, victor Tejfel

It is with great sadness that we have to report the death of Harold Masursky on August 24, 1990 at the age of 66 . Harold Masursky was the President of the working Group for Planetary System Nomenclature. He was well known for his active participation to space exploration of the planets. He was one of the pioneers of planetary geology. He wrote many important contributions on planetary surfaces.

\section{Introduction}

The physical study of planets and satellites is probably one of the more active fields of research of the second half of this century. Space exploration by automatic spacecrafts has completely renewed our knowledge of the solar system. The use of modern detectors, of large ground-based telescopes, and of powerful computers has considerably helped research. Planetary research (or planetology) is a pluridisciplinary domain, which requires not only the competence of astronomers, but also that of geophysicits, mineralogists, climatologists, biologists, chemists, physicists, "pure" mathematicians, and many other scientists. Many results are at the boundary of those of other commissions such as commissions $15,20,7,19,33,40,44,49$, and 51 .

The study of the main results obtained this last triennium shows a perfect complementarity between space and ground-based research. Both approaches have the same goals and are devoted to the study of the same objects. Quite often, the same individuals use both techniques, depending on the most efficient or on the more appropriate for the problem under study. It is remarkable to see that space data collected more than ten or fifteen years ago are still analized in connection with ground-based observations. The same remarks can apply for ground-based data. In addition to that, new theoretical models, new numerical simulations and new laboratory experiments have been recently developed. They all contribute to a better understanding of the physics of planets and satellites.

It is completely out of the scope of such a report to be exhaustive and to give credit to all active and efficient researchers. Instead of attempting the customary abbreviated 
summary, it seemed more appropriate to give here just a few examples in order to show how lively, how diverse, and how developed is planetary science. The examples quoted here have been taken more or less arbitrarily and are in no way the result of a selection and do not pretend to give an overview of the field. Advances in solar system studies are the consequence of the work of all the researchers who contribute to improve our understanding and not of only four or five outstanding articles as quoted in I.A.U. reports. The explosive increase of the number of published papers on planetary and satellite research has made it impossible to provide an adequate summary of progresses in the field over a given three year period in the few pages alloted for this purpose. Just the list of the titles of published papers should take much more room.

The period covered by this report, July 1987 to June 1990, witnessed a similar activity as the previous triennial interval. Due to the lack of space, it is a pitty to forget so many results, but the examples quoted here show the good health of this field of research.

Unfortunately, 1987 was the first year for 25 years where no new interplanetary spacecraft was launched in any country and no new planet fly-by or rendez vous done. Happily, space research is again quite active. One major event took place in 1989: the encounter of the voyager 2 spacecraft with the as yet poorly known Neptunian system. In spite of the failure of the Phobos spacecrafts well before the end of the mission, quite interesting results have been obtained on Phobos and Mars. The future of space exploration seems promising. The Magellan spacecraft started in 1990 a successful and spectacular analysis of Venus. The Galileo and Ulysse spacecraft have been successfully launched: their instruments are working well and should provide spectacular results during the nineties. Scientific teams of the Craf, Mars 94 and Cassini missions have been selected and these ambitious programs should be realized soon.

During this last triennium, another event played an important role: in many countries from Eastern Europe to chile, much more liberty was given to many scientists. For the first time, many colleagues were able to travel abroad from their native country and to participate to international meetings. This should rapidly improve the quality of science in these countries. It is a pitty that a meeting on Comparative Planetology scheduled in Nanking had been postponed from 1988 to 1989 and finally cancelled. We can only hope for the next triennium still more liberty for our colleagues! Most of the information can be found in journals such as Icarus, Space Science Reviews, Astronomy and Astrophysics, Soviet Astronomy, Nature, Science, and many others. More than one hundred books (technical as well as semipopular) have been published in several languages in the field of commission 16. Dozens of meetings have been organized during these past years. Among them, we may quote a conference devoted to the origin and evolution of atmospheres of the planets and their satellites, held in Tucson (Arizona) in March 1977 (Origin and Evolution of Planetary and Satellite Atmospheres, S.K. Atreya, J.B. Pollack, M.S. Matthews editors, University of Arizona Press, 1989), an International Mars 
conference held in Tucson (Arizona) in January 1988 (the proceedings will be published by the University of Arizona Press), the Uranus meeting held in Pasadena (California) in June 1988 (the proceedings will be published by the Univerity of Arizona Press), the first results of the Phobos mission announced in Paris (France) in october 1989 (the proceedings will be published in 1991) and the bioastronomy meeting held in Val Cenis (France) in June 1990 (the proceedings are in preparation).

Every year, the Division of Planetary Sciences of the American Astronomical society organizes a meeting where the latest results and discoveries are announced. About 400 planetologists of several countries are used to participate in these meetings. They were held in November 1987 in Pasadena (California), in November 1988 in Austin (Texas), in November 1989 in Providence (Rhode Island), in october 1990 in Charlottesville (Virginia). A good source of information can be found in the published abstracts (Bull. Amer. Astron. Soc., respectively vol. 19, p. 795-906; vol. 20, p.783-893; vol. 21 , p.895-1004, vol. 22, p. 1003-1140).

The preparation of this report was made possible through the kind cooperation of a number of colleagues. In particular, $T$. Owen, C. de Bergh, D. Gautier, B. Sicardy, F. Roques provided numerous helpful comments and suggestions.

\section{The Neptunian system}

One of the most important event in planetary science of this last years was the successful fly-by of Neptune by the Voyager 2 spacecraft in August 1989. In the course of encountering the Neptune system, some 9000 images were obtained. Observations were conducted in the visible, infrared, and ultraviolet as well as at centimeter wavelengths. The magnetic field was probed, plasmas were studied, energetic particles were measured, and radio emissions from Neptune were noted. The differential rotation of Neptune's upper atmosphere, the motion of spots, the great activity of Neptune's clouds and upper atmosphere andthe unexpected high winds have been analyzed. Six new satellites were discovered. The rings and arcs of Neptune discovered in 1984 and 1985 by A. Brahic, W. Hubbard and B. Sicardy by ground-based stellar occultation observations have been detected and imaged. This is a typical example of complementarity between ground-based and space observations. Thanks to the stellar occultation observations, Neptunian arcs have been detected and the Voyager 2 program has been changed in order to observe the Neptunian surroundings in good conditions. Thanks to the Voyager data, the overall structure of the rings and arcs have been understood. Thanks to the continuous observations from the ground, the stability of the arcs can be studied.

The very last voyager encounter was with Triton. It revealed a most interesting object. This cold and remote satellite seems surprisingly active. Triton's surface is not pock-marked with many impact craters. The density of Triton is about 2.08 , indicating a substantial rocky core. The atmosphere, consisting mainly of nitrogen, has a pressure of only about $10^{-5}$ that of the Earth. The surface apparently consists largely of water ice covered by solid 
nitrogen, which highly reflects sunlight. Surface temperature is about $38 \mathrm{~K}$. A small amount of methane present in the atmosphere is photodegraded to form $\mathrm{C}_{2} \mathrm{H}_{2}$ and other hydrocarbons. In spite of its $38 \mathrm{~K}$ surface temperature, Triton displays geyser-like activity. This was noted at the south polar region, which currently faces the sun. Presumably solar radiation penetrated into the solid nitrogen, causing greatly enhanced pressure and an eruption that carried any opaque material up to 8 kilometers above the surface.

Many years will be necessary to reduce most of the voyager data. Nethertheless, preliminary results have been presented in Science (vol. 246, p. 1417, 1989 and vol. 250, p. 410, 1990).

C. de Bergh, B. L. Lutz, T. Owen and J.P. Maillard (Astrophys. $J .331,453,1989$ ) have measured $\mathrm{D} / \mathrm{H}$ in Neptune's atmosphere from ground-based observations.

During the last ten years, more than one hundred stellar occultations by Neptune have been observed. Data are now systematically published (see for example: B. Sicardy, F. Roques and A. Brahic, Icarus, feb. 1991 or P.D. Nicholson, M.L. Cooke, K. Matthews, J.H. Elias and G. Gilmore, Icarus, 1991). Observational constraints and theoretical models of Neptune's arcs are reviewed by A. Brahic and W. Hubbard (Sky and Telescope 77, 605, 1989) and by J. Lissauer and P. Nicholson (Adv. Space Res. 10-1, 231, 1990). In order to understand the stability of the arcs and rings of Neptune, it would be necessary to continue to regularly observe ground-based stellar occultations.

\section{Uranus}

Data from the 1986 Voyager encounter have been extensively studied. In particular, a set of interesting papers have been published in the Journal of Geophysical Research (vol. 92, number A13, December 30, 1987). T.V. Johnson, R.H. Brown and J.B. Pollack (p. 14,884) have analyzed the densities of the Uranian satellites derived from Voyager data in order to place constraints on their uncompressed densities and probable silicate mass fractions. J. B. Plescia ( $p .14,918$ ) has presented data regarding Umbriel, Titania and oberon cratering records. B. Conrath, D. Gautier, R. Hanel, G. Lindal and $A$. Marten (p. 15,003) have measured the helium abundance. They found a value in good agreement with recent estimates of the solar helium abundance, suggesting that helium differentiation has not occurred on Uranus. F.M. Flasar, B.J. Conrath, P.J. Gierasch and J.A. Pirraglia (p. 15,011) have analyzed Voyager infrared observations in order to obtain Uranus'atmosphere thermal structure and dynamics.

P. Goldreich and C. Porco have analyzed the dynamics and the kinematics of Uranian rings (Astron. J. 93, 724, 1987) and have explored the rings-satellites resonances and interactions. French, Elliot, Kangas, Meech, and Holberg (Icarus 73, 349, 1988) have compared ground-based observations and Voyager 2 data in order to analyze Uranus' rings. Comparisons with ultraviolet occultations (Holberg, Nicholson, French and Elliot, Astron. J. 94, 178, 1987) and radio occultations (Gresh, Marouf, Tyler, Rosen and Simpson, Icarus $78,131,1989)$ have also been performed. 


\section{Jupiter and saturn}

An interesting book on time-variable phenomena in the Jovian system (NASA SP-494, 1989) has been recently published. It contains reviews on Io, Galilean satellites, Jupiter's rings, the atmosphere and the magnetosphere of Jupiter.

An excellent review of the composition of outer planet atmospheres has been done by $D$. Gautier and T. Owen (p. 487 in "Origin and Evolution of Planetary and Satellite Atmospheres", S.K. Atreya, J.B. Pollack, and M.S. Matthews editors, University of Arizona Press, 1989).

The vertical distribution of $C O$ in Jupiter's atmosphere was established by K.S. Noll, R.F. Knacke, T.R. Geballe and A.T. Tokunaga (Astrophys. J. 435, 1210, 1988). They were able to prove that $C O$ is present in Jupiter's troposphere by measuring the pressure broadening of individual $C O$ lines. This in turn demonstrates that the source of $\mathrm{CO}$ is the planet's interior, and not the infall of $\mathrm{H}_{2} \mathrm{O}$ containing particles from outside the planet. Therefore, the abundance of $\mathrm{CO}$ measured in the planet's troposphere can be used to set constraints on the global atmospheric oxygen abundance. Noll et al. found that the oxygen abundance on Jupiter must be close to the abundances of carbon and nitrogen. This is a very important result, since measurements of $\mathrm{H}_{2} \mathrm{O}$ had suggested a large oxygen deficiency on the planet. Noll, Geballe and Knacke (Astrophys. J. Lett. 338, L71, 1989) reported the detection of arsine on both Saturn and Jupiter, as did B. Bézard, P. Drossart, E. Lellouch, G. Tarrago and J.P. Maillard (Astrophys. J. 346, 509, 1989).

An excellent up-to-date review of Jupiter and Saturn interior models has been made by $W$. Hubbard and $M$. Marley (Icarus 78, 102, 1989) using recent accurate determinations of these planets' gravitational harmonics.

It has been envisaged for a long time that the giant planets could oscillate as the sun does. The Jovian oscillation pattern has been theoretically calculated (see for example B. Mosser, Icarus 87, 198, 1990). Seismology is in principle one of the most promising techniques to infer the internal structure of Jupiter and other giant planets. It seems that Jupiter pulsations have been detected for the first time using two different techniques (see for example F.X. Schmieder and B. Mosser, in Progress of seismology of the Sun and stars, Proc. Oji International Seminar, Hakone, Japan, Springer-Verlag, 1990).

Extensive studies of Titan have been made during these last years. T. Owen and D. Gautier (AdV. Space Res. 9, $\mathrm{n}^{\circ} 2,73,1989$ ) have reviewed the determination of abundances of minor constituents as a function of latitude and altitude.

A. Marten, D. Gautier, L. Tanguy, A. Lecacheux, C. Rosolen, and G. Paubert (Icarus 76, 558, 1988) have detected co in millimeter wavelengths in the troposphere and the stratosphere of Titan. L. Tanguy, B. Bézard, A. Marten, D. Gautier, E. Gérard, G. Paubert, and A. Lecacheux (Icarus 85, 43, 1990) have detected HCN on Titan from millimeter observations. C. de Bergh, B. L. Lutz, T. Owen and J. Chauville (Astrophys. J. 329, 951, 1988) have measured the $\mathrm{D} / \mathrm{H}$ ratio in Titan's atmosphere. 
The successful observation of the star 29 Sag. by Titan in July 1989 had led to spectacular results on the Titan's atmospheric temperature profile and on the chemical composition (B. Sicardy, A. Brahic et al., Nature 343, 350, 1990; W. Hubbard, D. Hunten et al., Nature 343, 349, 1990).

Many studies are still devoted to the study of saturn's rings and to the dynamics and the confinement of ring systems. One may quote for example the study of the mass segregation by J.M. Petit and M. Hénon (Astron. Astrophys. 188, 198,1988 and 199, 343, 1988), the simulations of colliding particles by Salo (Icarus $70,37,1987$ ) and Wisdom and Tremaine (Astron. J. 95, 925, 1988), the study of the confinement of sharp edges by $N$. Borderies, P. Goldreich and $S$. Tremaine (Icarus 80, 344, 1989).

\section{Pluto}

An occultation of a star by Pluto revealed its atmosphere and provided physical measurements (W.B. Hubbard, D.M. Hunten, S.W. Dieters, K.M. Hill, and R.D. Watson, Nature 336, 452, 1988; J.W. Elliot, E.W. Dunham, A.S. Bosh, S.M. Slivan, L.A. Young, L.H. Wasserman, and R.L. Millis, Icarus 77, 148, 1989). The essential data set is an occultation profile and a scale height. There is now extensive discussion about the validity of the assumption of an isothermal atmosphere and about possible haze layers. It seems likely that Pluto's atmosphere is an admixture of $\mathrm{CH}_{4}$ and $\mathrm{CO}$.

continued observations of the eclipses, transits and occultations of Charon increased the accuracy of mass and radius measurements and provided some information about albedo markings on Pluto's surface (see, for example, M.W. Buie and D.J. Tholen, Icarus 79, 23, 1989). Charon was found to be covered with water ice, and not methane.

After the surprising exploration of Triton, a comparison between the main physical characteristics and the atmospheric chemical composition of Pluto and Triton seems to indicate that pluto and Triton are members of the same family. Before the exploration of Pluto, probably during the next century, the best image of pluto is given by Triton.

\section{Mars}

A highlight of Martian studies during this triennium was the discovery and analysis of HDO in the Martian atmosphere (T. Owen, J.P. Maillard, C. de Bergh and B. Lutz, Science 240,1767, 1988; G. Bjoraker, M. Mumma, H.P. Larson, Bull. Am. Astron. Soc. 21, 990, 1989). Deuterium is enriched on Mars by about a factor of 5 compared with the Earth. The interpretation of this result in terms of the planet's original volatile inventory and the subsequent escape of both hydrogen and oxygen remains an open issue (Y.L. Yung, J.S. Wen, J.P. Pluto, M. Allen, and S. Paulson, Icarus 76, 146,1988 ).

According to D. Paige, D. Crisp and M. Santee (Bull. Am. Astron. Soc. 22, 1075, 1990), Mars, in common with the Earth, has snowstorms. These authors believe that Mariner 9 and Viking infrared data show clouds of frozen carbon dioxide high above the 
Martian poles in winter. If they are right, snowstorms trap dust and water ice on the poles and these deposits have recorded the history of Martian climate, much like the Earth's polar caps. The $\mathrm{D} / \mathrm{H}$ ratio has been detected on Mars by T. Owen, J.P. Maillard, C. de Bergh and B. Lutz (Science 240, 1767, 1988). The failure of the two Phobos spacecraft before completion of the mission is a tragedy, but the results of measurements made during the flight from Earth to Mars (52 days for Phobos 1 and 200 days for Phobos 2) as well as in the orbiting phase around Mars (57 days for Phobos 2) are quite interesting. Preliminary results have been published in Nature $(341,581,1989)$ and in the proceedings of the paris colloquium (see above). Mars surface has been probed by a gamma-ray spectrometer, an infrared thermal mapper and an infrared imaging spectrometer. New results have also been obtained on Phobos. In particular, the moon's density is about $1.95 \mathrm{~g} \mathrm{~cm}^{-3}$, which is somewhat less than determined by viking.

\section{Venus}

The most spectacular news about the Venus atmosphere during this triennium was the exploration of "windows" discovered in the spectrum of the planet's night-side at 1.74 and $2.35 \mathrm{~mm}$ by D. A. Allen and J.W. Crawford (Nature 307, 222, 1984). Thermal radiation escaping from the lower atmosphere allowed astronomers to photograph low-level clouds that are not visible in reflected sunlight (D.A. Allen, Icarus 69, 221, 1987; L.W. Kamp, F. Taylor, and S.B. Calcutt, Nature 336, 360, 1988; D. Crisp et al., Science $296,506,1989)$. This phenomenon has been exploited by the Galileo spacecraft during its Venus encounter in February 1990. It also opens up excellent opportunities for ground-based studies of the planet's lower atmosphere.

studies show Venus to be a dynamic planet whose surface is relatively young. An interesting review of the geology of Venus by A. Basilevsky and J. Head has been published in the Annual Review of Earth and Planetary Sciences $(16,295,1988)$. They review the high resolution data of Venera $15 / 16$.

The surface of Venus is successfully being probed by the Magellan spacecraft which started to observe in 1990. Despite the threat of technical problems, the spacecraft is routinely returning radar images with 120 meter resolution (ten times more precise than ever acquired before). At the end of 1990, only a small part of the surface has been explored showing spectacular impact craters, faults, volcanic craters, etc... The Magellan spacecraft is producing amazingly clear images which show a rich variety of features. Preliminary results have been announced at a meeting of the American Geophysical Union in December 1990.

\section{Mercury}

Mercury was explored by only one spacecraft: Mariner 10 in 1974 and 1975. Scientists are still reducing data from Mariner 10. A number of papers on the physics of Mercury have been published in Icarus (see, for example, a special issue of Icarus 71, 335, 1987). The proceedings of an international conference on Mercury have been 
published (F. Vilas, C. Chapman, and M. Matthews, editors, University of Arizona Press, 1988).

\section{The Moon}

The origin of the Moon is still an open problem. For centuries, lunar origin myths have usually centered around three possibilities (fission, capture, and binary accretion). Since the origin of the Moon conference held in Hawaii in october 1984 (Hartmann, Phillips, and Taylor, editors, NASA Lunar and Planetary Institute, Houston, Texas, 1986), the collision hypothesis has been studied by many authors. Efforts have been made to simulate giant impacts. An interesting review of the collision Hypothesis has been made by D. Stevenson in the Annual Review of Earth and Planetary Sciences $(15,271,1987)$. More work is needed and it is not yet clear whether the collision hypothesis satisfies the observational facts.

\section{The origin and the dynamics of the solar system}

The problem of the origin of the solar system is still very lively. Several excellent review papers have been recently published. A.G.W. Cameron discussed various recent developments in the Annual Review of Astronomy and Astrophysics (26, 441, 1988). J. Lunine (Icarus 81, 1, 1989) reviewed the volatile processes in the outer solar system. The idea that regions around the forming giant planets were physically and chemically distinct from the solar nebula gains some credence from data on bulk densities and compositions. D. Gautier (Phil. Trans. Roy. Soc. London A325, 583, 1988) has reviewed the constraints on the formation of giant planets from their atmospheric chemical composition. J. Lissauer (Icarus 69, 249, 1987) has presented an unified scenario of planetary accretion in which he gives timescale of formation and details on the structure of the protoplanetary disc. One can also quote the study of a model of accumulation of a swarm of small planetesimals by G.W. Wetherill and G.R. Stewart (Icarus 77, 330, 1989) and the study of the accretion rates of protoplanets by $Y$. Greenzweig and $J$. Lissauer (Icarus 87, 40, 1990).

Recent discoveries of nonlinear dynamics have open a new and rapidly growing field of research. It is now well-known that the phase space of most Hamiltonian systems is divided: for some initial conditions, the trajectories are quasi-periodic, for some others, they are chaotic. Even if the solar system is generaliy perceived as evolving with clockwork regularity, it is just a dynamical system which presents sometimes a chaotic behaviour. There are several physical situations where chaotic solutions of Newton's equations play an important role (Hyperion chaotic rotation, orbital history of irregularly shaped satellites, Kirkwood gaps, long term evolution of Pluto's orbit, etc...). A review of these problems by $J$. Wisdom can be found in Icarus (72, $241,1987)$.

André BRAHIC

President of the commission 